Research Article

\title{
Electrospun Core-Shell Hollow Structure Cocatalysts for Enhanced Photocatalytic Activity
}

\author{
Fengyun Guo $\mathbb{D}^{1},{ }^{1}$ Ziyi Guo, ${ }^{1}$ Lei Gao, ${ }^{1}$ Dongming Qi, ${ }^{1}$ Guichu Yue, ${ }^{2}$ Nü Wang, ${ }^{2}$ Yong Zhao, ${ }^{2}$ \\ Ni Li, ${ }^{1}$ and Jie Xiong ${ }^{1}$ \\ ${ }^{1}$ Key Laboratory of Advanced Textile Materials and Fabrication Technology of Ministry of Education, School of Textile Science and \\ Engineering (International Silk Institute), Zhejiang Sci-Tech University, Hangzhou 310018, China \\ ${ }^{2}$ Key Laboratory of Bioinspired Intelligent Interface Science and Technology, Ministry of Education, School of Chemistry, \\ Beihang University, Beijing 100191, China)
}

Correspondence should be addressed to Fengyun Guo; guofy@zstu.edu.cn

Received 1 April 2021; Revised 20 April 2021; Accepted 9 May 2021; Published 25 May 2021

Academic Editor: Jim Low

Copyright (C) 2021 Fengyun Guo et al. This is an open access article distributed under the Creative Commons Attribution License, which permits unrestricted use, distribution, and reproduction in any medium, provided the original work is properly cited.

The core-shell $\mathrm{NaYF}_{4} / \mathrm{Yb} / \mathrm{Tm} / \mathrm{TiO}_{2}$ hollow composite fibers were prepared by coaxial electrospinning and high-temperature calcination. The composite fibers exhibit excellent photocatalytic activity under the dual synergistic of regulating the core-shell hollow microstructure and the composition by doping nanoparticles. Compared with commercial P25 and hollow fiber without nanoparticles, the degradation efficiency of rhodamine B using the core-shell composite fiber was significantly improved up to 99\%. Moreover, the nanoparticles in the composite fibers can exist stably and maintain good structure and photocatalytic activity after repeated use. Therefore, the composite fiber has a wide application prospect in photocatalytic degradation of organic pollutants.

\section{Introduction}

Water pollution is one of the most serious problems all mankind faced. Providing and sustainably managing water and sanitation for all is one of the sustainable development goals. The UN World Water Development Report proposes to improve waste water management by reducing source pollution, removing waste water pollutants, recycling recycled water, and recovering useful byproducts. With the development of scitech and society, global attention to water pollution control is increasing year by year. Since Fujishima and Honda discovered that $\mathrm{TiO}_{2}$ could be used as an electrode for photodegradation of aquatic hydrogen in 1972 [1], the development of new $\mathrm{TiO}_{2}$-based photocatalytic materials and their application to degradation of organic dyes and other pollutants has become a worldwide research hotspot [2-5].

Due to the excellent photocatalytic activity, chemical stability, environmentally friendly feature, and low biological toxicity of $\mathrm{TiO}_{2}, \mathrm{TiO}_{2}$-based catalyst is currently recognized as the most promising photocatalyst for organic dye degrada- tion and has great potential in wastewater purification treatment $[6,7]$. However, due to the wide intrinsic band gap of $\mathrm{TiO}_{2}$, it can only absorb ultraviolet light and has a low utilization rate of sunlight. At the same time, the carrier life of $\mathrm{TiO}_{2}$ is short, and the photogenerated electrons and holes are easy to compound, leading to the degradation of catalytic performance [8]. Recombining $\mathrm{TiO}_{2}$ and other nanomaterials, making composite catalyst absorbance range extension to visible light area, and further improving the efficiency of separation and migration of the carrier, is the main research direction for developing high-performance $\mathrm{TiO}_{2}$ composite photocatalyst. The current main composite methods generally include cationic replace, precious metal nanoparticle load, and heterostructure built. $\mathrm{Li}$ et al. [9] prepared $\mathrm{V}^{3+} / \mathrm{V}^{5+}$ substituted mesoporous $\mathrm{TiO}_{2}$ using the sol-gel method for catalytic degradation of methylene blue. Compared with mesoporous $\mathrm{TiO}_{2}$, vanadium ion substituted mesoporous $\mathrm{TiO}_{2}$ has higher photocatalytic activity. Zhang et al. [10] reported $\mathrm{HAc}-\mathrm{Ag} / \mathrm{TiO}_{2}$ composite photocatalyst loaded with silver nanoparticles using the photochemical 


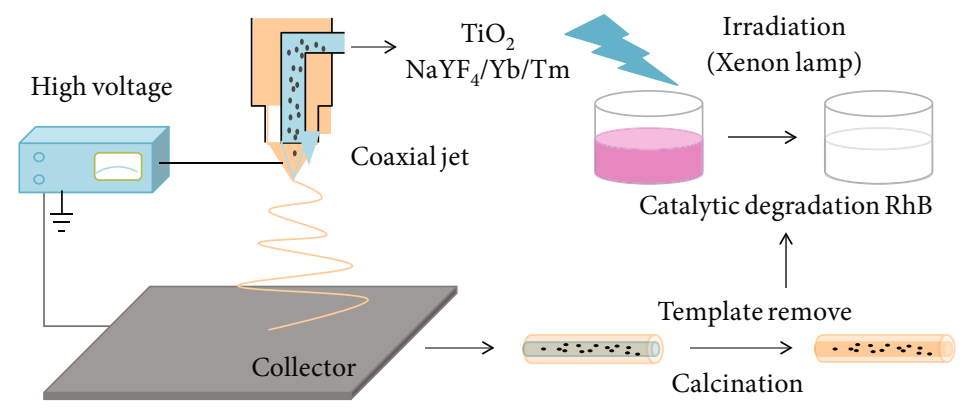

FIGURE 1: Electrospun core-shell $\mathrm{TiO}_{2}$ nanofibers with incorporated $\mathrm{NaYF}_{4} / \mathrm{Yb} / \mathrm{Tm}$ nanoparticles. The inner cyclohexane and paraffin were removed by postcalcination treatment resulting in $\mathrm{TiO}_{2}$ tubes containing nanoparticles. Subsequently, the composite nanofibers were used to catalyze rhodamine B degradation.

reduction method combined with acetic acid impregnation for the degradation of methyl orange in water. The synergy of $\mathrm{HAc}$ and $\mathrm{Ag}$ makes $\mathrm{HAc}-\mathrm{Ag} / \mathrm{TiO}_{2}$ a more narrower band gap width and correction to the top of valence band location, which endow a good visible light catalytic activity. Building heterojunction is widely applied in catalysis. For example, Wang et al. [11] deposited $\mathrm{Ag}_{2} \mathrm{O}$ on $\mathrm{TiO}_{2}$ nanorods constructing the $\mathrm{Ag}_{2} \mathrm{O} / \mathrm{TiO}_{2}$ heterojunction. $\mathrm{Ag}_{2} \mathrm{O}$ has a narrower band gap than $\mathrm{TiO}_{2}$, and the electron-hole pair generated under visible light irradiation is captured by the electron trap of $\mathrm{TiO}_{2}$, which prolongs the recombination time of electrons and holes, and realizes the efficient catalytic degradation of methyl orange in water.

The core-shell structure has the advantages of large specific surface area, regular size, stable, and controllable performance, which makes it a wide application prospect in the field of catalysis [12-14]. Greiner et al. [15] prepared the core-shell structure fiber of gold nanoparticles supported on polymer tube by the electrospinning technique. The fiber has obvious catalytic activity for the hydrolysis oxidation and alcoholysis of dimethylphenylsilane. Moreover, the macroscopic nonwoven fabric can be used as the catalyst system and shows excellent reusable performance. In recent years, upconversion nanomaterials have become a research hotspot due to the characteristics of near-infrared photoexcitation, narrow emission band, large antistoke shift, stable photochemical properties, and adjustable luminescence properties [16-19]. Through the multiphoton process, the upconversion material doped with rare earth elements can convert low energy near-infrared light to high energy visible light and ultraviolet light and stimulate $\mathrm{TiO}_{2}$ to complete the photocatalytic effect. It is one of the effective means to improve the utilization of light by compound the upconversion material with the ultraviolet photocatalytic material to broaden the intrinsic excitation wavelength of the photocatalytic material. The upconversion materials can be doped with rare earth elements to further regulate and improve their luminescence properties.

Based on the above considerations, a $\mathrm{NaYF}_{4} / \mathrm{Yb} / \mathrm{Tm} /-$ $\mathrm{TiO}_{2}$ core-shell fiber was designed and prepared by the coaxial electrospinning method and liquid paraffin wax as a position holder. $\mathrm{NaYF}_{4} / \mathrm{Yb} / \mathrm{Tm}$ can convert near-infrared light and visible light to ultraviolet light and be utilized by $\mathrm{TiO}_{2}$ for the degradation of rhodamine $\mathrm{B}$ in waste water.

\section{Experimental Part}

2.1. Reagents and Instruments. Reagents include polyvinylpyrrolidone (PVP, Mw = 1300000, Aldrich), anhydrous ethanol (AR, Beijing Chemical Factory), glacial acetic acid (AR, Beijing Chemical Factory), tetrabutyl titanate (AR, National medicine group chemical reagent Co., Ltd.), rhodamine $B$ (red dye, Aldrich), liquid paraffin wax (AR, Beijing Chemical Factory), and cyclohexane (AR, Beijing Chemical Factory), and $\mathrm{NaYF}_{4} / \mathrm{Yb} / \mathrm{Tm}$ nanoparticles are given as a present from the University of Wollongong. The experimental instruments include electrospinning device (high voltage power supply, receiving device, and syringe), scanning electron microscopy (SEM, Quanta 250 FEG), transmission electron microscope (TEM, FEI Tecnai G2-F20), muffle furnace (German, LH15/13), and UV-Vis spectrophotometer (Hitachi U3900).

\subsection{Experimental Process}

2.2.1. The Preparation of Precursor Solution. (1) The shell spinning solution: dissolving $0.5 \mathrm{~g}$ PVP in a mixed solution of $7.5 \mathrm{~g}$ anhydrous ethanol and $2.0 \mathrm{~g}$ acetic acid and continuous stirring it with a magnetic agitator to form a homogeneous and clarified solution until PVP is fully dissolved. Dissolving $2.0 \mathrm{~g}$ tetrabutyl titanate in the above mixture, stirring it slowly with a magnetic stirrer during the adding process, and continue stirring for $1 \mathrm{~h}$ after forming a homogeneous solution. The solution was evenly mixed resulting in PVP/tetrabutyl titanate precursor solution. (2) The core spinning solution: referring to the method in the literature [20], $\mathrm{NaYF}_{4} / \mathrm{Yb} / \mathrm{Tm}$ nanoparticles were synthesized by solvothermal technology, and the optimal ratio of core spinning solution was determined. And then $300 \mathrm{~L}$ of nanoparticle dispersion was measured, and $200 \mathrm{~L}$ of cyclohexane and $3.5 \mathrm{~mL}$ of paraffin were added to prepare core spinning solution for use.

\subsubsection{Preparation of $\mathrm{NaYF}_{4} / \mathrm{Yb} / \mathrm{Tm} / \mathrm{TiO}_{2}$ Core-Shell Hollow} Structure Composite Fibers. The electrospinning process was performed using a home-made setup including coaxial needles and basic equipment. The inner and outer layer precursor body solutions were feed into the syringe, respectively, and the diameter, voltage, and receiving distance of the needle were controlled in the coaxial electrospinning process. 


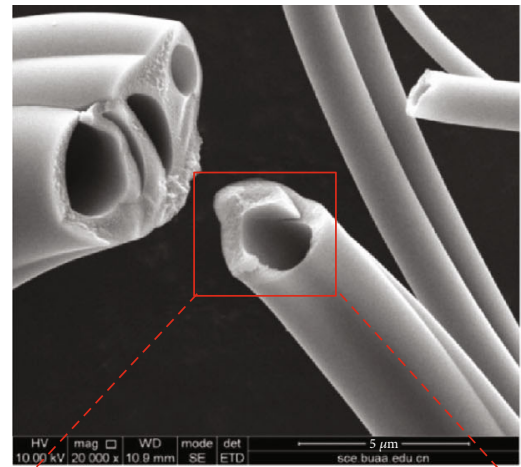

(a)

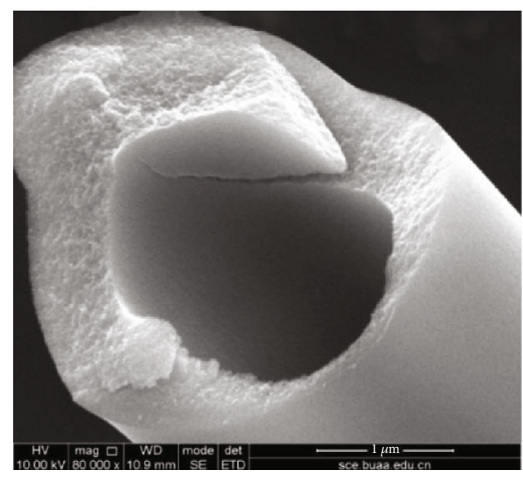

(d)

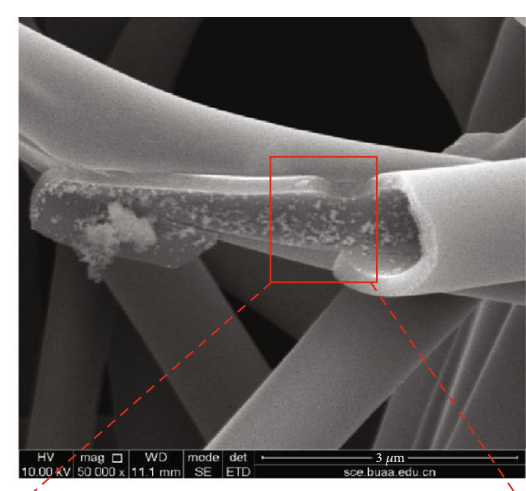

(b)

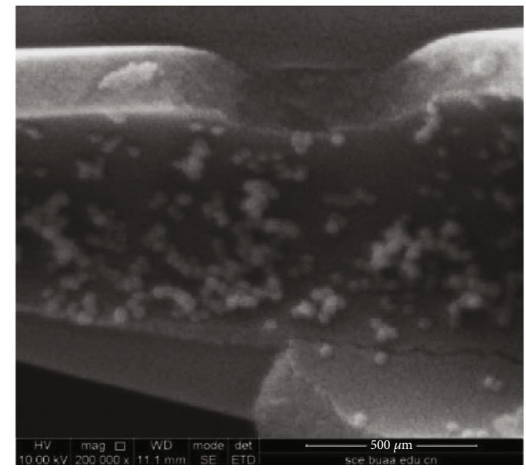

(e)

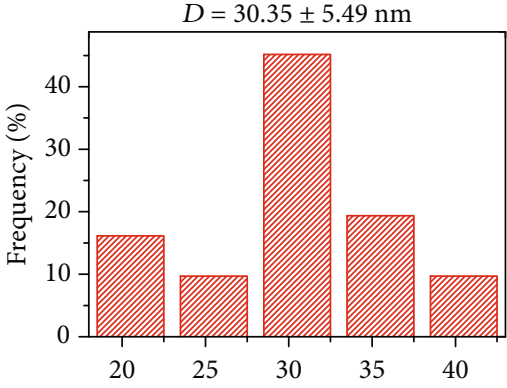

(c)

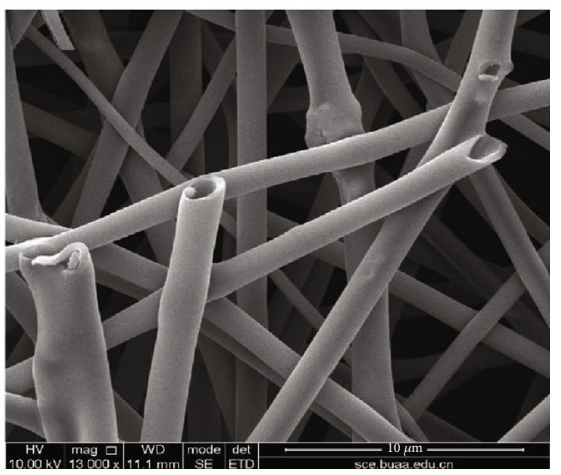

(f)

FIGURE 2: Morphology characterization and diameter distribution of nanoparticle and nanofibers. (a, b) Cross-sectional SEM images of TiO ${ }_{2}$ and $\mathrm{NaYF}_{4} / \mathrm{Yb} / \mathrm{Tm} / \mathrm{TiO}_{2}$ core-shell hollow fiber $(c, d)$ proving the existence of $\mathrm{NaYF}_{4} / \mathrm{Yb} / \mathrm{Tm}$ nanoparticles on the inner surfaces. (e, f) Size statistic of nanoparticles and SEM image of core-shell fibers.
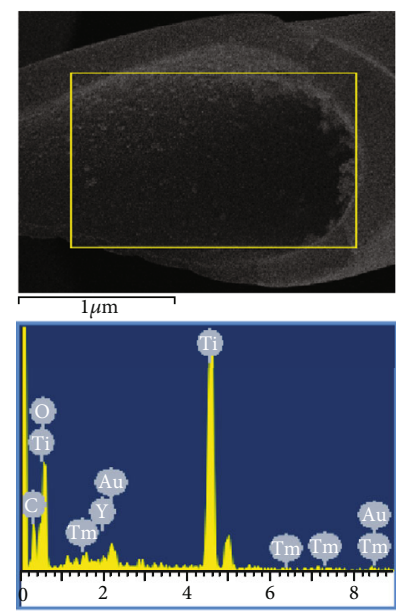
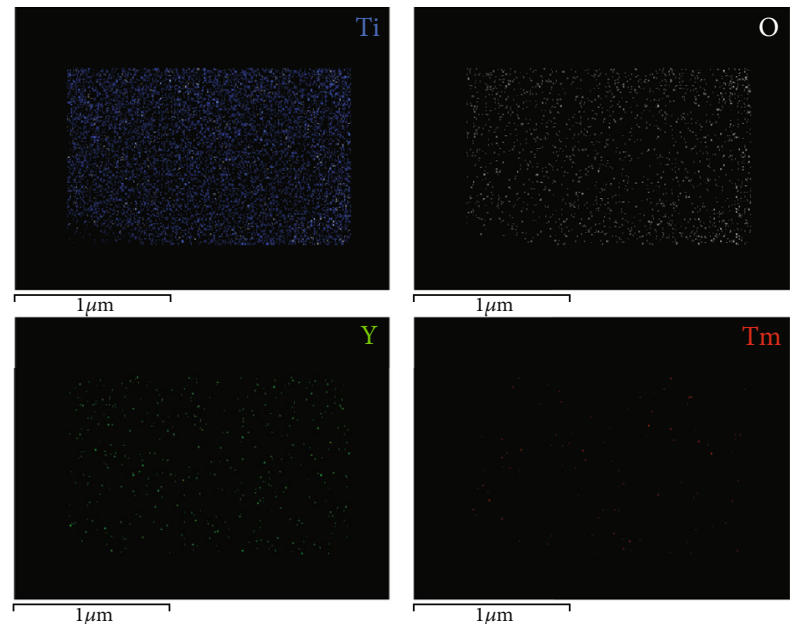

Figure 3: EDS showing the distribution of element.

Nanoparticle/PVP/tetrabutyl titanate primary fiber membrane was obtained by adjusting the solution parameters and electrospinning conditions. Next, the primary fiber membrane was placed in a temperature-controlled muffle furnace at a heating rate of $1^{\circ} \mathrm{C} / \mathrm{min}$, calcined at $500^{\circ} \mathrm{C}$ in air atmosphere, and naturally cooled to room temperature. The organic components were removed, and the nanoparti- cles were coated in the interior surface of fibers obtaining the core-shell hollow composite fiber membrane.

2.2.3. Morphology Characterization. The morphology characterization was performed by the following procedure using different techniques. For the SEM sample, the fiber membrane and its cross-sectional were directly pasted on the 


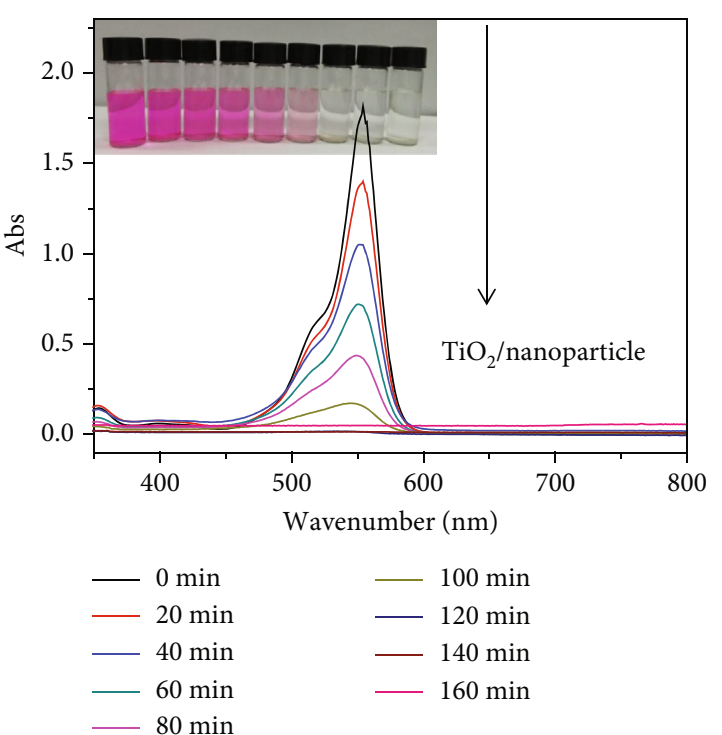

(a)

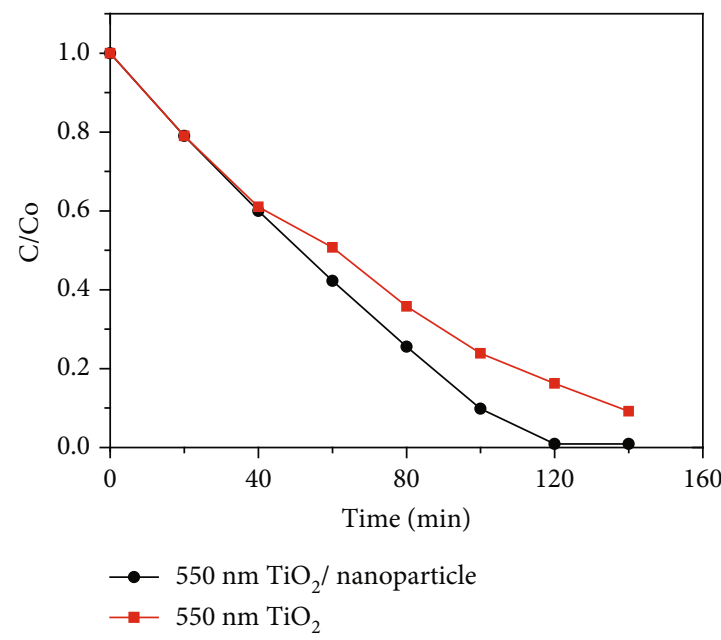

(c)

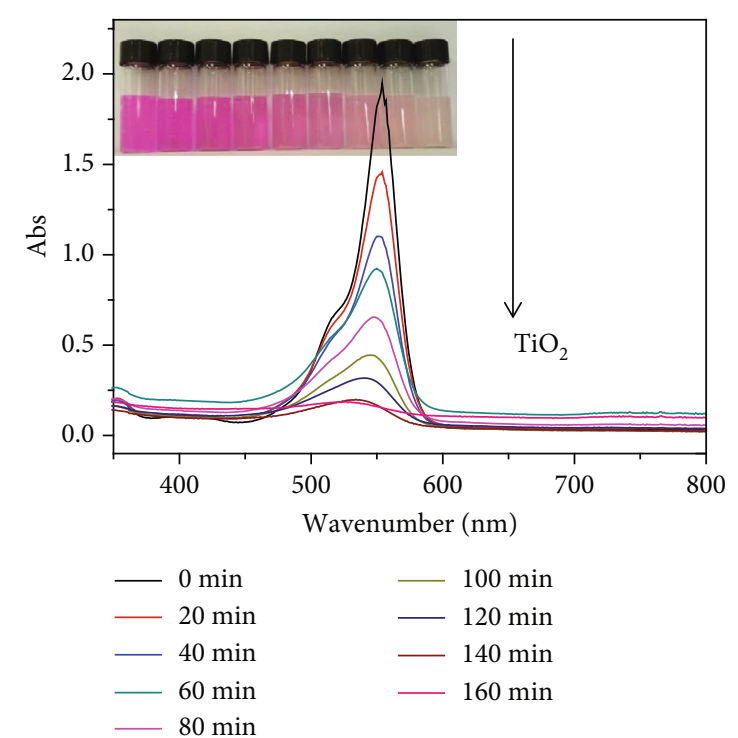

(b)

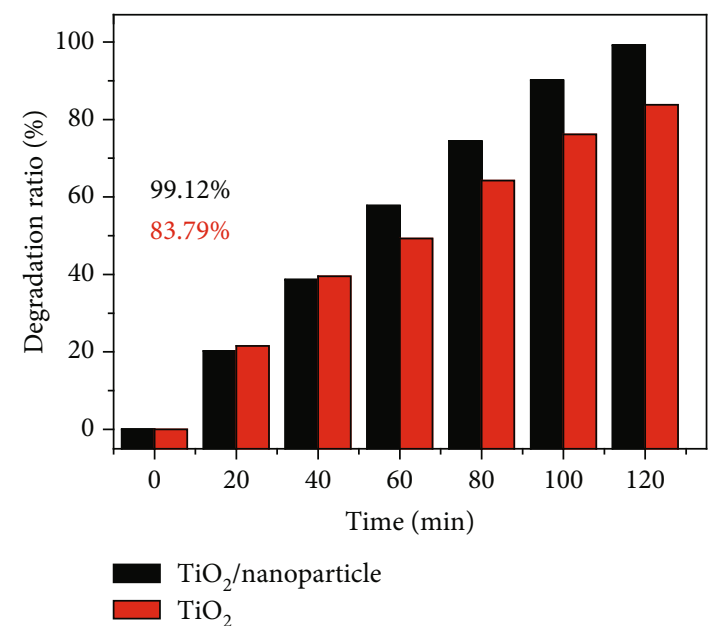

(d)

Figure 4: Catalytic activity of core-shell fibers. ( $a, b)$ UV/Vis absorption spectrum of the reaction solution with different reaction times for photodegradation of rhodamine $\mathrm{B}$ with $\mathrm{TiO}_{2}$ /nanoparticle and $\mathrm{TiO}_{2}$ core-shell nanofiber sample. (c, d) Concentration changes and degradation ratios of rhodamine $\mathrm{B}$ with and without nanoparticle of $\mathrm{TiO}_{2}$.

SEM test platform, gold was sprayed under vacuum condition, and then the surface morphology and section structure of the fiber were characterized by SEM. The acceleration voltage was $10 \mathrm{kV}$. For TEM samples, nanoparticles and calcined samples were dispersed in ethanol solution, dropped on the surface of copper mesh with carbon film, and dried naturally, and then the morphology of the nanoparticles and the internal structure of the fibers were characterized by TEM. The acceleration voltage was $200 \mathrm{kV}$. Finally, the size of the nanoparticles was statistical and obtained from one hundred selected samples.

2.2.4. Photocatalytic Degradation Experiment. The photocatalytic properties of composite fibers were characterized by catalytic degradation of rhodamine B. The composite fiber membrane obtained from the calcination was cut into small pieces and placed in the prepared rhodamine B solution, which was soaked under the condition of avoiding light to achieve saturated adsorption. The sample to be tested was placed flat in a surface dish containing $10 \mathrm{~mL}$ and $0.01 \mathrm{mmol} / \mathrm{L}$ rhodamine B solution, irradiated with xenon lamp, and the supernatant fluid was taken into a quartz colorimetric dish at a certain interval of time. The absorbance at $554 \mathrm{~nm}$ corresponds to the absorption maximum of rhodamine B. The absorbance value was measured by UV-Vis spectrophotometer to describe the concentration change and examine its photocatalytic activity. The test results were obtained from five selected samples. For comparison, the photocatalytic experiments of the two types of core-shell hollow structure fiber with and without $\mathrm{NaYF}_{4} / \mathrm{Yb} / \mathrm{Tm}$ nanoparticles were carried out under the same conditions. Quantification of the rhodamine B concentration at different 


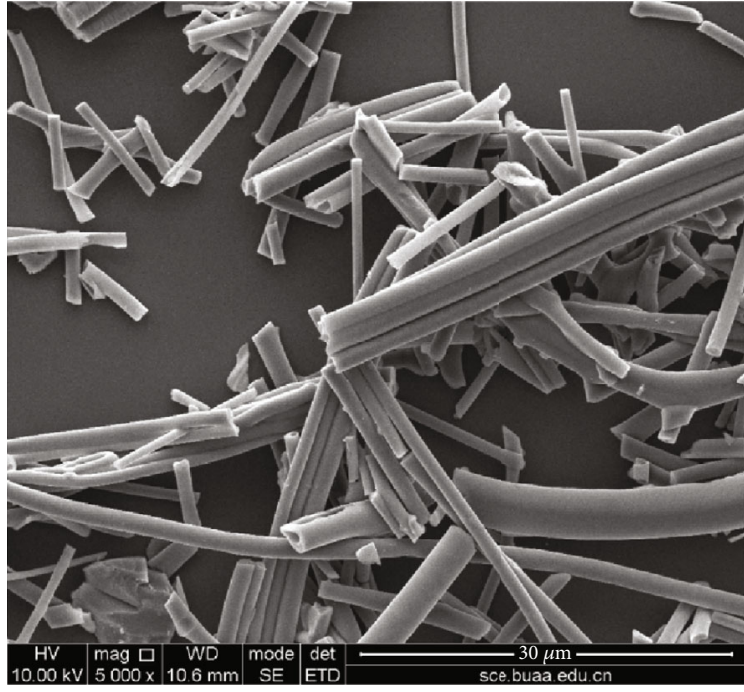

(a)

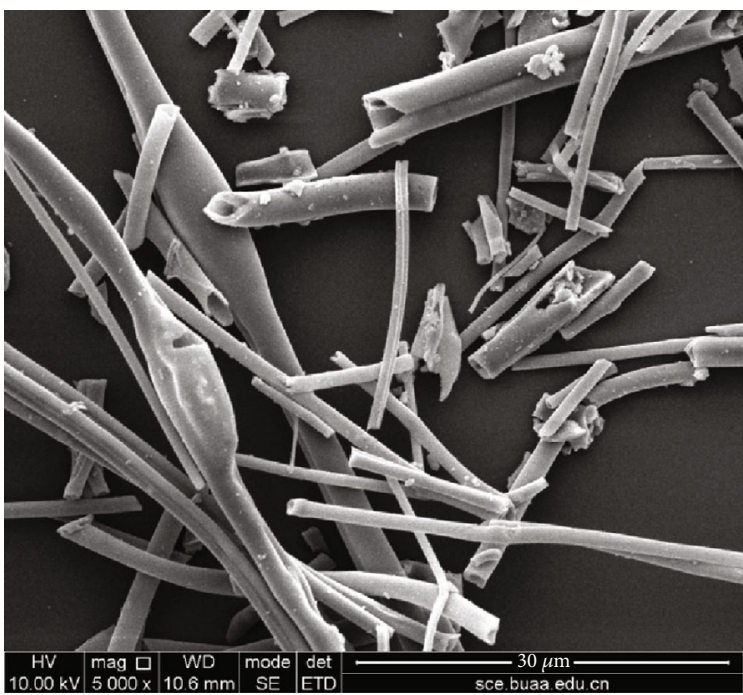

(c)

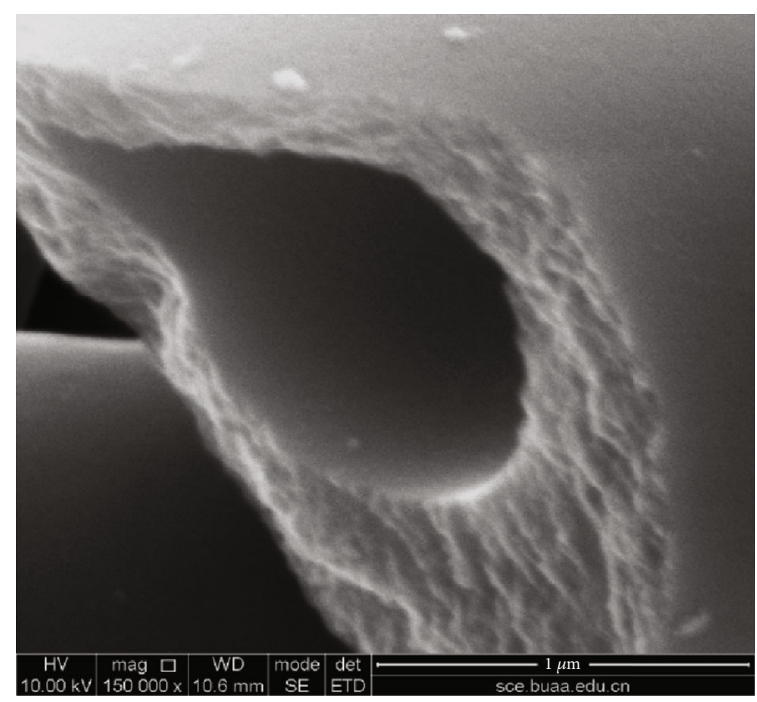

(b)

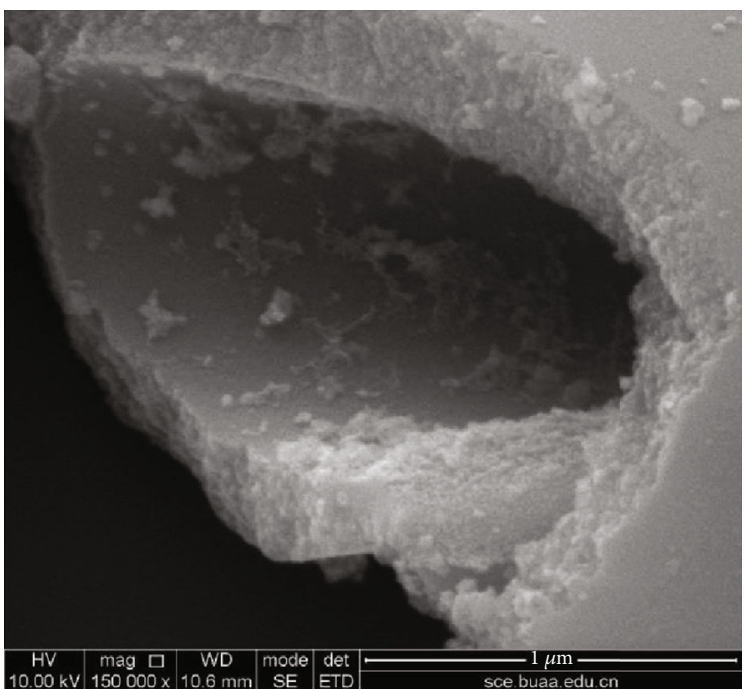

(d)

FIGURE 5: Morphology of nanofibers after catalytic degradation. (a, b) Surface appearance of $\mathrm{TiO}_{2}$ (a) and NaYF$/ \mathrm{Nb}_{4} / \mathrm{Tm} / \mathrm{TiO} \mathrm{O}_{2}(\mathrm{~b})$ core-shell hollow fiber proving almost intact morphology. (c, d) Random cross-sectional images of $\mathrm{TiO}_{2}$ (c) and $\mathrm{NaYF}_{4} / \mathrm{Yb} / \mathrm{Tm} / \mathrm{TiO}{ }_{2}(\mathrm{~d})$ core-shell hollow fiber proving the apparent existence of $\mathrm{NaYF}_{4} / \mathrm{Yb} / \mathrm{Tm}$ nanoparticles after catalytic degradation.

intervals was taken by using a calibration plot of rhodamine B. The absorbance was measured every 20 minutes until the color did not change significantly, and then the photocatalytic degradation curve of the material under light conditions was plotted.

\section{Results and Discussion}

3.1. Preparation, Morphology, and Structure Analysis of $\mathrm{NaYF}_{4} / \mathrm{Yb} / \mathrm{Tm} / \mathrm{TiO}_{2}$ Core-Shell Composite Fibers. As shown in Figure 1, we first prepared core-shell hollow $\mathrm{TiO}_{2}$ nanofibers with and without incorporated $\mathrm{NaYF}_{4} / \mathrm{Yb} / \mathrm{Tm}$ nanoparticles using coaxial electrospinning and calcination and then investigated catalytic degradation of rhodamine B. Specifically, two types of core-shell hollow structure fiber with $\mathrm{NaYF}_{4} / \mathrm{Yb} / \mathrm{Tm}$ nanoparticles and without $\mathrm{NaYF}_{4} / \mathrm{Yb} / \mathrm{Tm}$ nanoparticles were selected for comparison. The fibers were obtained by coaxial electrospinning with optimized preparation parameters and calcination at $500^{\circ} \mathrm{C}$, and their surface and fracture morphology and structure were characterized and analyzed using SEM and TEM. Figure 2 shows the corresponding scanning electron microscope images and statistical images of particle diameter. Figures 2(b) and 2(d) are local enlargements of Figures 2(a) and 2(c), respectively. After calcination, interior cyclohexane and paraffin are removed, leaving only the hollow structure or the nanoparticles. As can be seen from Figures 2(a)-2(d), the core-shell hollow $\mathrm{TiO}_{2}$ fiber has smooth interior surface, showing an obvious hollow core-shell structure. As can be seen from Figures 2(c) and 2(d), after adding nanoparticles, the surface of the fiber is smooth, but the interior of the fiber is obviously covered with nanoparticles. The size of the prepared 


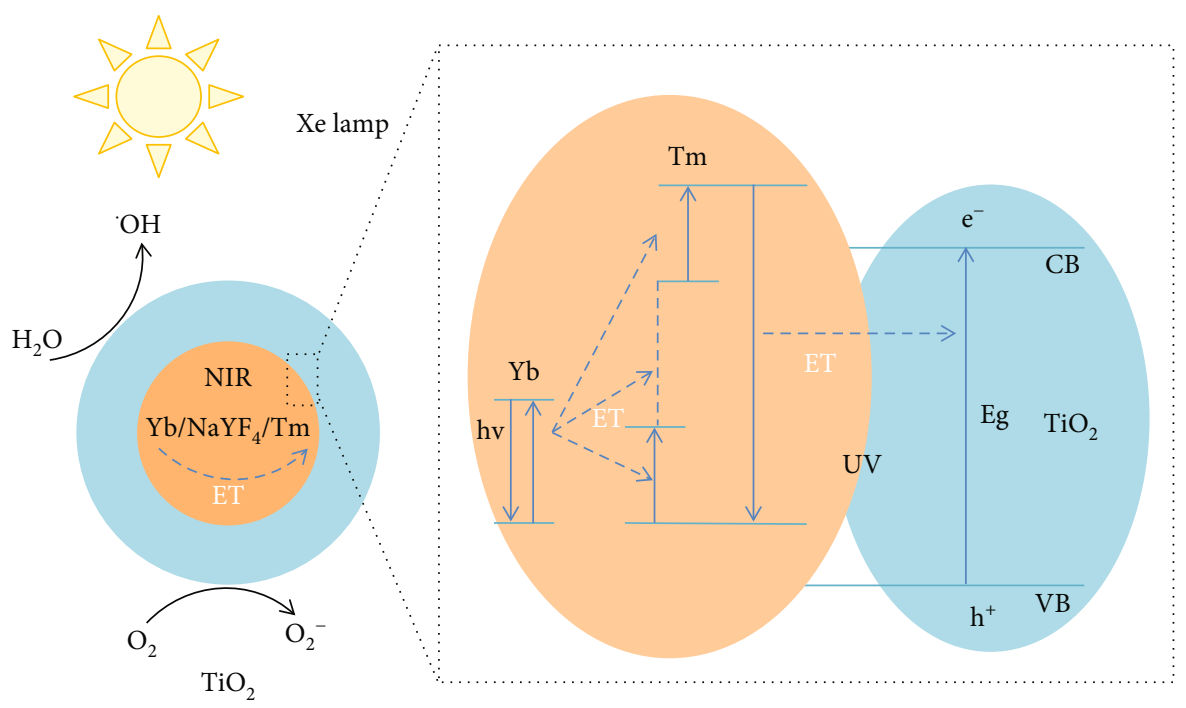

FIgURE 6: Mechanism analysis about the energy transfer process between $\mathrm{NaYF}_{4} / \mathrm{Yb} / \mathrm{Tm}$ and $\mathrm{TiO}_{2}$ and the generation of $\cdot \mathrm{OH}$ and $\mathrm{O}_{2}{ }^{-}$.

nanoparticles is small and uniform. According to statistics, the average particle size of the nanoparticles is $30.35 \pm 5.49$ $\mathrm{nm}$, as shown in Figure 2(e). For the overall morphology, fibers cross each other in a state of disordered accumulation, as shown in Figure 2(f). The EDS element analysis diagram in Figure 3 also proves that the composite fiber successfully loads nanoparticles.

3.2. Performance Analysis of Photocatalytic Degradation of Rhodamine $\mathrm{B}$ by $\mathrm{NaYF}_{4} / \mathrm{Yb} / \mathrm{Tm} / \mathrm{TiO}_{2}$ Core-Shell Composite Fibers. In order to prove the photocatalytic degradation performance of composite fibers, the catalytic degradation experiment of rhodamine B was carried out, and the results were shown in Figure 4. The composite fiber catalyst was first added to the as-prepared rhodamine B solution and stirred for $30 \mathrm{~min}$ to achieve adsorption equilibrium with rhodamine B. After stirred, the nanoparticles were naked. Then, the sample was placed in a colorimetric dish, and the UVVis absorption spectrum was measured in the wavelength range of 300-800 $\mathrm{nm}$. Figures 4(a) and 4(b) show the absorption spectra at different catalytic times. At $550 \mathrm{~nm}$, the maximum absorption peak intensity gradually decreases with the extension of reaction time. As can be seen from the illustration, as time goes on, the catalytic degradation reaction proceeds, and the color of the dye changes from dark to light until it totally disappears. However, in the absence of nanoparticles, the catalytic degradation rate was slower, indicating that both nanoparticles and structures are vital factors for the catalysis. After $2 \mathrm{~h}$ reaction, the degradation efficiency of fiber without nanoparticles was $83.79 \%$, while the degradation efficiency of fiber with nanoparticles was $99.12 \%$. The catalytic degradation efficiency of both of them was much higher than the photocatalytic degradation rate of commercial $\mathrm{TiO}_{2}$ (P25) $[16,17]$. These results indicate that the composite fiber has excellent photocatalytic activity, in which the synergistic effect of the hollow core-shell structure and nanoparticles plays an important role in improving the photocatalytic performance.
3.3. Cyclic Utilization Analysis of $\mathrm{NaYF}_{4} / \mathrm{Yb} / \mathrm{Tm} / \mathrm{TiO}_{2}$ CoreShell Composite Fibers. In order to prove the recyclability, the fiber morphology before and after photocatalysis was characterized, as shown in Figure 5. It can be found that the morphology and internal structure of the fiber membrane have no obvious change before and after photocatalysis, the core-shell structure of the fiber was almost intact, the internal covered nanoparticles still existed, and the loading effect was unsacrificed. In addition, as a catalyst carrier, the own chemical stability and thermal stability of the core-shell micro/nanofiber make it difficult to be degraded by ultraviolet light or strong oxidizing hydroxyl radicals in the long-term photocatalytic process, indicating that the fiber have good photocatalytic performance and loading stability.

3.4. Photocatalytic Mechanism Analysis of $\mathrm{NaYF}_{4} / \mathrm{Yb} / \mathrm{Tm} / \mathrm{TiO}_{2}$ Core-Shell Composite Fibers. As shown in Figure 6, for $\mathrm{NaYF}_{4} / \mathrm{Yb} / \mathrm{Tm} / \mathrm{TiO}_{2}$ core-shell fiber catalyst, the possible photocatalytic reaction mechanism is shown as follows: NaY$\mathrm{F}_{4} / \mathrm{Yb} / \mathrm{Tm}$ absorbs visible light and converts it into ultraviolet light and emits it to $\mathrm{TiO}_{2}$, photogenerated electrons migrate to the surface of $\mathrm{TiO}_{2}$ and react with adsorbed oxygen to generate $\mathrm{O}^{2-}$ or $\mathrm{O}_{2}{ }^{2-}$, and then $\mathrm{O}^{2-}$ or $\mathrm{O}_{2}{ }^{2-}$ reacts with $\mathrm{H}_{2} \mathrm{O}$ to generate $\mathrm{H}_{2} \mathrm{O}_{2}$ and $\cdot \mathrm{OH}$. The conduction band of $\mathrm{TiO}_{2}$ is above the REDOX potential of rhodamine $\mathrm{B}$; so, the oxygen-containing active species $\left(\mathrm{O}^{2-}, \mathrm{O}_{2}{ }^{2-}, \mathrm{H}_{2} \mathrm{O}_{2}, \cdot \mathrm{OH}\right.$, etc.) produced by photoexcitation of $\mathrm{TiO}_{2}$ have the performance of oxidizing organic molecules.

\section{Conclusion}

$\mathrm{NaYF}_{4} / \mathrm{Yb} / \mathrm{Tm} / \mathrm{TiO}_{2}$ core-shell composite fibers were prepared by coaxial electrospinning and high temperature calcination. The morphology, structure, and photocatalytic properties of the materials were characterized by comprehensive techniques including scanning electron microscopy, transmission electron microscopy, and UV-Vis spectrophotometer. By dual synergistic, the degradation efficiency of 
rhodamine B using the core-shell composite fiber was significantly improved up to $99 \%$, which is superior to commercial P25 and hollow fiber without nanoparticles. The results show that the design of the hollow core-shell structure and the doping of upconversion nanoparticles can greatly improve the photocatalytic performance of the composite fiber, and the material has a potential application prospect in the catalytic degradation of organic pollutants.

\section{Data Availability}

The data used to support the findings of this study are included within the article.

\section{Conflicts of Interest}

The authors declare that they have no conflicts of interest.

\section{Acknowledgments}

This work is supported by the National Natural Science Foundation of China, Grant 51803183.

\section{References}

[1] A. Fujishima and K. Honda, "Electrochemical photolysis of water at a semiconductor electrode," Nature, vol. 238, no. 5358, pp. 37-38, 1972.

[2] M. R. Hoffmann, S. T. Martin, W. Choi, and D. W. Bahnemann, "Environmental applications of semiconductor photocatalysis," Chemical Reviews, vol. 95, no. 1, pp. 69-96, 1995.

[3] X. B. Chen and S. S. Mao, "Titanium dioxide nanomaterials: synthesis, properties, modifications, and applications," Chemical Reviews, vol. 107, no. 7, pp. 2891-2959, 2007.

[4] Y. N. Tang, W. di, X. S. Zhai, R. Y. Yang, and W. P. Qin, "NIRresponsive photocatalytic activity and mechanism of $\mathrm{NaY}$ F4:Yb,Tm@TiO2core-shell nanoparticles," ACS Catalysis, vol. 3, no. 3, pp. 405-412, 2013.

[5] H. W. Lin, K. Zhang, G. L. Yang et al., "Ultrafine nano 1T$\mathrm{MoS}_{2}$ monolayers with $\mathrm{NiO}_{\mathrm{x}}$ as dual co-catalysts over $\mathrm{TiO}_{2}$ photoharvester for efficient photocatalytic hydrogen evolution," Applied Catalysis. B, Environmental, vol. 279, p. 119387, 2020.

[6] A. L. Linsebigler, G. Q. Lu, and J. T. Yates, "Photocatalysis on TiO2 surfaces: principles, mechanisms, and selected results," Chemical Reviews, vol. 95, no. 3, pp. 735-758, 1995.

[7] S. Furukawa, T. Shishido, K. Teramura, and T. Tanaka, "Photocatalytic oxidation of alcohols over TiO2covered with Nb2O5," ACS Catalysis, vol. 2, no. 1, pp. 175-179, 2012.

[8] C. Zhang, D. Huang, M. Sun et al., "Promoting effect of nonmetal ion doping and hierarchically 3D dendrimeric architecture for visible-light-active mesoporous TiO2 photocatalyst," Chemical Journal of Chinese Universities, vol. 38, no. 3, pp. 471-478, 2017.

[9] P. Xu, Y. Li, C. Liu, M. Li, and R. Deng, "Preparation and visible-light photocatalytic performance of mesoporous vanadium-doped titania," Chemical Journal of Chinese Universities, vol. 35, no. 9, pp. 1945-1961, 2014.

[10] A. Shan, J. Zhen, J. Lui, J. Bai, J. Yang, and Q. Zhang, "Preparation and synergistic catalytic performance of $\mathrm{Ag} / \mathrm{TiO} 2 \mathrm{com}-$ posite photocatalysts modified by acetic acid," Chemical
Journal of Chinese Universities, vol. 38, no. 8, pp. 1450-1457, 2017.

[11] W. Wang, J. He, F. Cui, and C. Wang, "Preparation of Ag2O/$\mathrm{TiO} 2$ nanowires heterojunction and their photocatalytic activity under visible-light irradiation," Chemical Journal of Chinese Universities, vol. 36, no. 7, pp. 1367-1371, 2015.

[12] W. Hao, T. Zhai, X. Wang, and T. Wang, "Fabrication and Catalytic Activity of Nickel Core-shell Structure," Chemical Journal of Chinese Universities, vol. 31, no. 6, pp. 1213-1217, 2010.

[13] G. C. Yue, S. Li, D. M. Li et al., "Coral-like Au/TiO2hollow nanofibers with through-holes as a high-efficient catalyst through mass transfer enhancement," Langmuir, vol. 35, no. 14, pp. 4843-4848, 2019.

[14] J. Tian, Y. F. Zhang, L. du et al., "Tailored self-assembled photocatalytic nanofibres for visible-light-driven hydrogen production," Nature Chemistry, vol. 12, no. 12, pp. 1150-1156, 2020.

[15] F. Mitschang, H. Schmalz, S. Agarwal, and A. Greiner, "Teabag-like polymer nanoreactors filled with gold nanoparticles," Angewandte Chemie International Edition, vol. 53, no. 19, pp. 4972-4975, 2014.

[16] C. C. Wang, K. L. Song, Y. Feng et al., "Preparation of NaLuF4:Gd, Yb, Tm-TiO2nanocomposite with high catalytic activity for solar light assisted photocatalytic degradation of dyes and wastewater," RSC Advances, vol. 4, no. 74, pp. 39118-39125, 2014.

[17] Q. L. Ye, X. Yang, C. Li, and Z. Li, "Synthesis of UV/NIR photocatalysts by coating $\mathrm{TiO} 2$ shell on peanut-like YF3:Yb,Tm upconversion nanocrystals," Materials Letters, vol. 106, no. 1, pp. 238-241, 2013.

[18] J. Zhao, D. Jin, E. P. Schartner et al., "Single-nanocrystal sensitivity achieved by enhanced upconversion luminescence," Nature Nanotechnology, vol. 8, no. 10, pp. 729-734, 2013.

[19] C. Lee, E. Xu, Y. Liu et al., "Giant nonlinear optical responses from photon-avalanching nanoparticles," Nature, vol. 589, no. 7841, pp. 230-235, 2021.

[20] N. V. Golubko, M. I. Yanovskaya, I. P. Romm, and A. N. Ozerin, "Hydrolysis of titanium alkoxides: thermochemical, electron microscopy, saxs studies," Journal of Sol-Gel Science and Technology, vol. 20, no. 3, pp. 245-262, 2001. 